\title{
Probiotic therapy reduces inflammation and improves intestinal morphology in rats with induced oral mucositis
}

\author{
Dayana GERHARD(a) \\ Frederico José da Silva Simão de \\ SOUSA ${ }^{(a)}$ \\ Rodrigo Antonio Carvalho \\ ANDRAUS ${ }^{(b)}$ \\ Paulo Eduardo PARDO(c) \\ Gisele Alborguetti NAI(d) \\ Hermann Bremer NETO(e) \\ Michel Reis MESSORA ${ }^{(f)}$ \\ Luciana Prado MAIA(g)
}

(a) Universidade do Oeste Paulista Unoeste, School of Dentistry, Presidente Prudente, SP, Brazil.

(b) Universidade Norte do Paraná - Unopar, Rehabilitation Sciences Program, Londrina, PR, Brazil.

(c) Universidade do Oeste Paulista - Unoeste, Department of Veterinary Medicine, Presidente Prudente, SP, Brazil (retired)

(d) Universidade do Oeste Paulista - Unoeste, Department of Pathology, Presidente Prudente, SP, Brazil.

(e)Universidade do Oeste Paulista Unoeste, Department of Functional Sciences, Presidente Prudente, SP, Brazil.

(f) Universidade de São Paulo - USP, School of Dentistry of Ribeirão Preto, Ribeirão Preto, SP, Brazil.

(g) Universidade do Oeste Paulista Unoeste, Graduate Program in Dentistry, Presidente Prudente, SP, Brazil.

Declaration of Interests: The authors certify that they have no commercial or associative interest that represents a conflict of interest in connection with the manuscript.

Corresponding Author:

Luciana Prado Maia

E-mail: lucianapmaia@gmail.com

https://doi.org/10.1590/1807-3107BOR-2017.vol31.0071

Submitted: Feb 08, 2017

Accepted for publication: May 22, 2017

Last revision: June 07, 2017

\begin{abstract}
The aim of the present study was to evaluate the effect of systemic administration of probiotics (PROB) on the progression of experimentally induced oral and intestinal mucositis in rats immunosuppressed by chemotherapy (5-fluorouracil: 5-FU). Twenty-four rats were divided into the following groups $(n=6)$ : GC (control), GPROB, G5FU and G5-FU/PROB. Groups GPROB and G5-FU/PROB received $1 \mathrm{~g}$ of probiotic incorporated into each $100 \mathrm{~g}$ of feed (Bacillus subtilis, Bifidobacterium bifidum, Enterococcus faecium and Lactobacillus acidophilus), beginning 30 days before oral mucositis induction. Groups G5FU and G5-FU/PROB received $60 \mathrm{mg} / \mathrm{kg}$ of 5 -FU on days 0 and 2. The left oral mucosa of each animal was irritated by mechanical trauma (days 1 and 2). On days 3 and 7, three animals from each group were sacrificed, and their oral mucosa and small intestine were biopsied and processed for histopathological analysis. Groups G5-FU and G5-FU/PROB showed ulcerated oral lesions at day 3, with progression in group G5-FU and regression in group G5-FU/PROB at day 7. Histologically, less severe signs of inflammation in the oral mucosa were observed in group G5-FU/PROB than in group G5-FU. Regarding the intestine, villus-related defects of lesser magnitude were observed in group G5-FU/PROB, compared with group G5-FU. Group GPROB showed greater villus height than group GC. It can be concluded that probiotic supplementation reduced oral and intestinal inflammation in immunosuppressed rats with experimentally induced mucositis, and may protect the intestine from changes induced by chemotherapy, thus contributing to overall health.
\end{abstract}

Keywords: Drug Therapy; Intestines; Mucositis; Stomatitis; Probiotics

\section{Introduction}

Radiotherapy and chemotherapy are the primary methods used for cancer treatment. Among the most widely used drugs in cancer chemotherapy, 5-fluorouracil (5-FU) ${ }^{1}$ may cause cytotoxicity not limited to neoplastic cells, but also affecting normal tissues, ${ }^{2}$ because it produces reactive oxygen, which damages the tissue and initiates a cascade of biological events, ultimately damaging the DNA of epithelial basal cells and causing cell death. ${ }^{3}$ Immunosuppression from radiotherapy and chemotherapy causes adverse reactions in the oral cavity, such as oral mucositis, xerostomia, dysphagia, dysgeusia, candidiasis, and radiodermatitis. ${ }^{4}$ 
Oral mucositis (OM) is the most common clinical complication observed in oral cancer treatment. The pathogenesis of OM has not been fully explained, but mechanismsinvolving theoral mucosa, pro-inflammatory cytokines and local factors, such as saliva and microbiota, have been suggested as playing a role in this process. ${ }^{5,6}$ There is also a potential interaction between the oral microenvironment and the development of mucositis. OM is clinically associated with mucosal changes ranging from erythema to ulcerative lesions in different locations of the oral mucosa, commonly located on non-keratinized surfaces, such as the jugal mucosa, center and edges of the tongue, soft palate and floor of the mouth. Because $\mathrm{OM}$ is also associated with constant, intense pain, it generates great discomfort, may restrict food intake and speech, and is often a site of bleeding and infection, as well as a systemic portal of entry for oral bacteria. OM leads to decreased quality of life, sleep disorders and poor oral hygiene, and may delay or interrupt cancer treatment. ${ }^{8} \mathrm{OM}$ treatment consists of locally or systemically administered pharmacological agents, such as anesthetics, analgesics, and antimicrobials. Other treatment options, such as cryotherapy and laser treatment, have also been used extensively. ${ }^{9}$ However, none of these methods has proved effective or been widely accepted as a standard treatment. ${ }^{10}$

5-FU can also cause intestinal mucositis by inducing apoptosis of intestinal stem cells, ${ }^{11}$ resulting in villus blunting and crypt shortening ${ }^{12}$ reduced mucosal cell DNA, RNA and protein content, ${ }^{13,14,15}$ and reduced brush border enzyme activity. ${ }^{12}$ Intestinal mucositis is associated with abdominal pain and bloating, intestinal ulceration, nausea, vomiting, diarrhea, constipation, anorexia, dehydration and intestinal and systemic infections. ${ }^{16,17}$ Treatment of intestinal mucositis is similar to that of oral mucositis: antibiotics administered to prevent intestinal infection and bacteremia; however, the downside is that they may allow opportunistic resistant pathogenic bacteria to colonize the intestine. ${ }^{18}$

Traditionally, probiotics (PROB) have been associated with intestinal health, and greater clinical interest for PROB has focused on either preventing or treating gastrointestinal infections and diseases. Recently, the literature has suggested their use as a treatment approach to intestinal mucositis. ${ }^{19,20,21,22,23}$ However, over the last decade, an increasing number of established effects and proposals for probiotic bacteria have been reported, including increased adaptive immune response, treatment or prevention of infections in respiratory and urogenital tracts, and prevention or mitigation of allergies and atopic diseases in infants. ${ }^{24,25}$ The mechanisms by which probiotics may contribute to the prevention and treatment of certain pathologies include direct interaction with or competitive exclusion of other microorganisms, and modulation of the host's immune system. ${ }^{26}$ The oral cavity has just recently been suggested as an important target for probiotic application. ${ }^{27}$ Until recently, probiotics were assessed mainly for the purpose of controlling dental caries, by potentially reducing the levels of Streptococcus mutans in the saliva. ${ }^{28,29,30}$ Regarding periodontal disease, studies have shown that probiotic supplementation reduces alveolar bone loss in rats with ligature-induced periodontitis. ${ }^{26,31}$

Considering that changes in immunological and inflammatory responses, and in the microbiota, are factors involved in the development of mucositis, the use of probiotics undoubtedly opens a new and promising avenue for the preventive treatment of this disease, and well-controlled studies are valuable to structure the future pillars of probiotic therapy. In this context, the present study aimed at assessing the effect of the systemic administration of probiotics on the progression of experimentally induced oral and intestinal mucositis in rats immunosuppressed by chemotherapy.

\section{Methodology}

This study was initiated and performed after approval from the Ethics Committee on Animal Use (Protocol n. 2163) of the University of Western São Paulo (UNOESTE).

Twenty-four male Wistar rats (Rattus norvegicus albinus) with a mean body mass of $150 \mathrm{~g}$ were used. They were kept in individual cages and under the same standard conditions of illumination (light/dark cycle of $12 / 12$ hours), controlled temperature of $22 \pm 1^{\circ} \mathrm{C}$, and food and water ad libitum. The animals were randomly divided into the following experimental groups:

a. GC: Control group, rats with no chemotherapy or probiotic administration $(n=6)$;

b. GPROB: rats with probiotic administration, but no chemotherapy administration $(n=6)$; 
c. G5-FU: rats with chemotherapy administration, but no probiotic administration $(n=6)$;

d. G5-FU/PROB: rats with chemotherapy and probiotic administration $(n=6)$.

Groups GPROB and G5-FU/PROB received $1 \mathrm{~g}$ of probiotic incorporated into each $100 \mathrm{~g}$ of feed (Bacillus subtilis - $2.9 \times 10^{8} \mathrm{UFC} / \mathrm{g}$, Bifidobacterium bifidum - 2.0 $\times 10^{8} \mathrm{UFC} / \mathrm{g}$, Enterococcus faecium - $2.1 \times 10^{8}$ $\mathrm{UFC} / \mathrm{g}$, Lactobacillus acidophilus - $1.0 \times 10^{8} \mathrm{UFC} / \mathrm{g}$ ). This incorporated supplementation was initiated 30 days before mucositis induction. The feed consumption of all the experimental groups was evaluated weekly.

Immunosuppression was induced using the method described by Sonis et al. ${ }^{32}$ and modified by Mitsuhashi et al. ${ }^{33}$ The animals received two intraperitoneal injections of $5-\mathrm{FU}(60 \mathrm{mg} / \mathrm{kg})$, administered at days 0 and 2 of the experiment.

To mimic the clinical effect of local irritation and induce mucosal ulceration, the mucosa was irritated by mechanical trauma (MT), following a protocol adapted from Sonis et al. ${ }^{32}$ This was performed by anesthetizing the animals with an intraperitoneal injection of $2 \%$ xylazine hydrochloride solution ( $2 \mathrm{mg} / \mathrm{mL}$ ) (Rompum - Bayer Saúde Animal) and $10 \%$ ketamine hydrochloride $(10 \mathrm{mg} / \mathrm{mL}$ ) (Dopalen Agribrands do Brasil Ltda). After general anesthesia, the left jugal mucosa was inverted and irritated by MT, performed by dragging the tip of an 18-gauge needle with a $100 \mathrm{~mm}^{2}$ area in a vertical and horizontal linear pattern along the inverted mucosa, at days 1 and 2 of the experiment.

Animal conditions were evaluated by measuring feed consumption weekly, and assessing weight at baseline and days $0,1,2,3$ and 7 . The data were expressed as body weight change in relation to initial weight at baseline.

The animals were anesthetized at days 3 and 7, and standardized photographs of the inverted jugal mucosa were obtained for macroscopic analysis. Inflammatory aspects such as erythema, hyperemia, hemorrhagic areas, epithelial ulcerations and abscesses were analyzed descriptively in the images obtained.

Three animals from each group were euthanized at day 3 , and the 3 remaining animals were euthanized at day 7. Euthanasia was performed by intraperitoneal injection of thiopental (Thiopentax, Cristália, Brazil) at a dose of $100 \mathrm{mg} / \mathrm{kg}$.

\section{Histopathological analysis of the ulcerated area}

Samples of the jugal mucosa were removed from the euthanized animals. The specimens were fixed in $10 \%$ buffered formalin solution, dehydrated and embedded in paraffin. Sections ( $5 \mu \mathrm{m}$-thick) were obtained for hematoxylin and eosin (H\&E) staining and light microscopy examination (NIKON Labophot, Japan). The parameters of inflammatory cell infiltration and vascular dilatation, and the presence of hemorrhagic areas, edema, ulcerations, and abscesses were classified into scores ranging from 0 to 3 , according to the method reported by Lima et al..$^{34}$

0 : normal epithelium and connective tissue with no vasodilation; absence of or discreet cellular infiltration; absence of hemorrhagic areas, ulcerations or abscesses.

1: discreet vascular ingurgitation; areas of re-epithelialization; discreet inflammatory infiltration with mononuclear prevalence; absence of hemorrhagic areas, edema, ulcerations or abscesses.

2: moderate vascular ingurgitation; areas of hydropic epithelial degeneration, inflammatory infiltration with neutrophil prevalence; presence of hemorrhagic areas, edema and eventual ulcerations; absence of abscesses.

3: severe vascular ingurgitation and dilatation; inflammatory infiltration with neutrophil prevalence; presence of hemorrhagic areas, edema and extensive ulcerations and abscesses.

\section{Histomorphometric intestinal analysis}

Samples of small intestines were collected and fixed in $10 \%$ buffered formalin solution for 48 hours, routinely processed and incorporated with paraffin. The sections obtained ( $5 \mu \mathrm{m}$ thick) were stained with H\&E for light microscopy analysis. Histomorphometric analysis was performed by obtaining 3 favorably orientated sections selected from each animal, cutting perpendicularly from the villus enterocytes to the muscularis mucosa. Images of the histological sections were captured by a digital camera connected to a light microscope. The digital images were analyzed using appropriate software (Image J 1.50b, National Institutes of Health, USA). The villus height $(\mathrm{VH})$ was obtained by measuring 
the vertical distance from the villus-crypt junction to the lower limit of the crypt, in 10 crypts per animal. The $\mathrm{VH}$ values for each animal were represented by the mean values of the three histological sections.

\section{Statistical Analysis}

The quantitative data obtained were grouped according to the experimental group and presented as mean and standard deviation. The Mixed Model for Repeated Measures was used to compare the weight and feed consumption among the groups. Kruskal-Wallis was used to compare the groups according to the histological score of the oral mucosa. Intestinal histomorphometric analysis was performed using the ttest for intergroup comparisons and the paired ttest for intragroup comparisons. A 5\% significance level was adopted for all the statistical analyses. The SPSS version 21 (IBM Software, Armonk, NY, USA) was the statistics software used for these analyses.

\section{Results}

\section{Clinical Follow-up}

Regarding the animals' weight, there was no statistically significant difference among the groups at baseline and days $0,1,2$, and 3 . At day 7 , group GC presented a weight gain $(326.67 \pm 28.57 \mathrm{Kg})$, followed by GPROB $(293 \pm 34.18 \mathrm{Kg}), \mathrm{G} 5-\mathrm{FU} / \mathrm{PROB}(245.5 \pm 33.23 \mathrm{Kg})$, and G5-FU $(241.67 \pm 22.9 \mathrm{Kg})$, with a statistically significant difference between group GC and groups G5-FU and G5-FU/PROB ( $\mathrm{p}=0.016)$ (Figure 1A). Regarding feed consumption, measured weekly, there was no significant difference among the groups, at any of the experimental time points (Figure 1B).

Photographic images representing each group and the experimental time points are shown in Figure 2. The macroscopic analysis showed discreet ulceration in groups GC and GPROB present at day 3, but absent at day 7. At day 3, groups G5-FU and G5-FU/PROB presented a single whitish ulcerative lesion of irregular shape and with elevated edges, moderate erythema and hyperemia. At day 7 , group G5-FU showed a well-delimited lesion having severe erythema and hyperemia, and having increased in size from day 3 to 7. Group G5-FU/PROB presented a reduction in the ulcerative lesion from day 3 to 7 , with no erythema or hyperemia.

\section{Histopathological analysis of the ulcerated area of the jugal mucosa}

The histopathological analysis of the jugal mucosa for group GC at day 3 showed inflammatory infiltration and occasional mucosal ulcerations covered by a fibrinleukocyte crust exhibiting a prevalence of moderate inflammation (Figure 3A); however, at day 7, most samples presented full mucosal re-epithelialization and discreet submucosal inflammatory infiltration (Figure 3B). Group GPROB showed unchanged epithelium at day 3 (Figure 3C) and day 7 (Figure 3D). All the samples of group G5-FU presented moderate vascular ingurgitation,

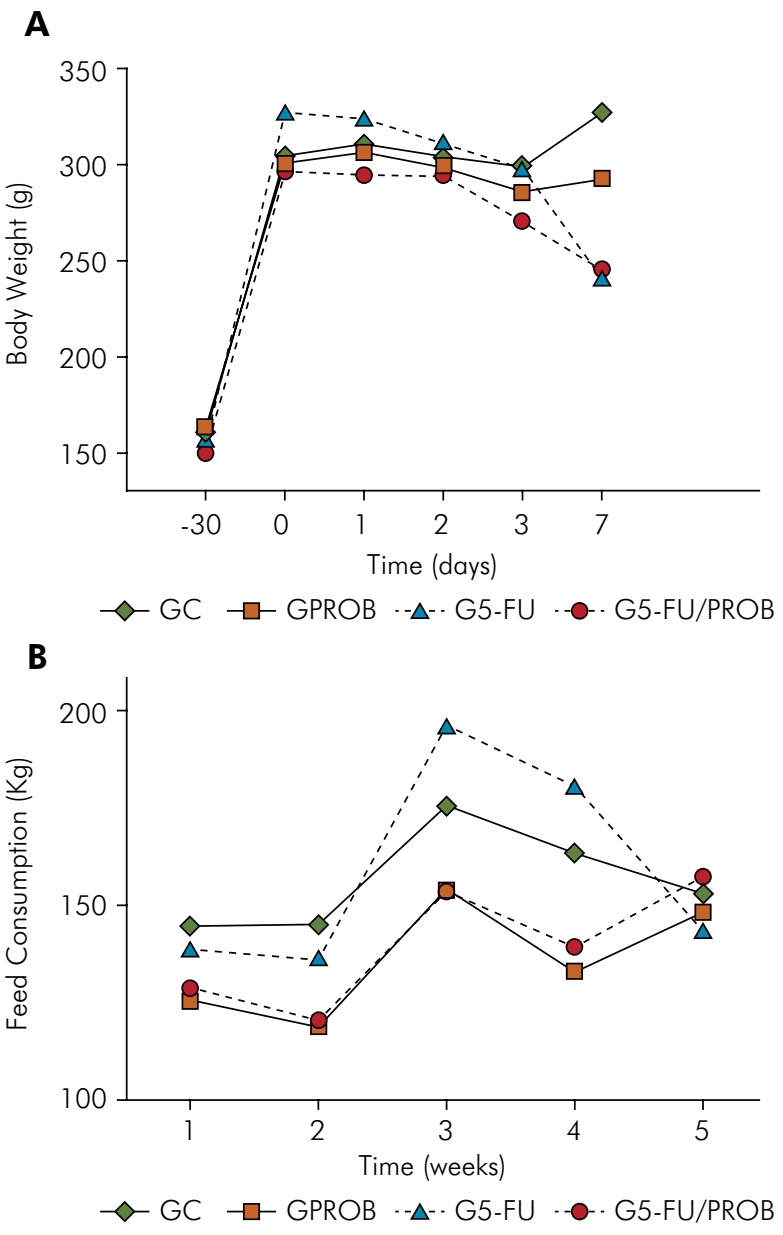

Figure 1. A: Body weight variation of all groups throughout the experimental period; $\mathrm{B}$ : Feed consumption variation of all the groups throughout the experimental period. 

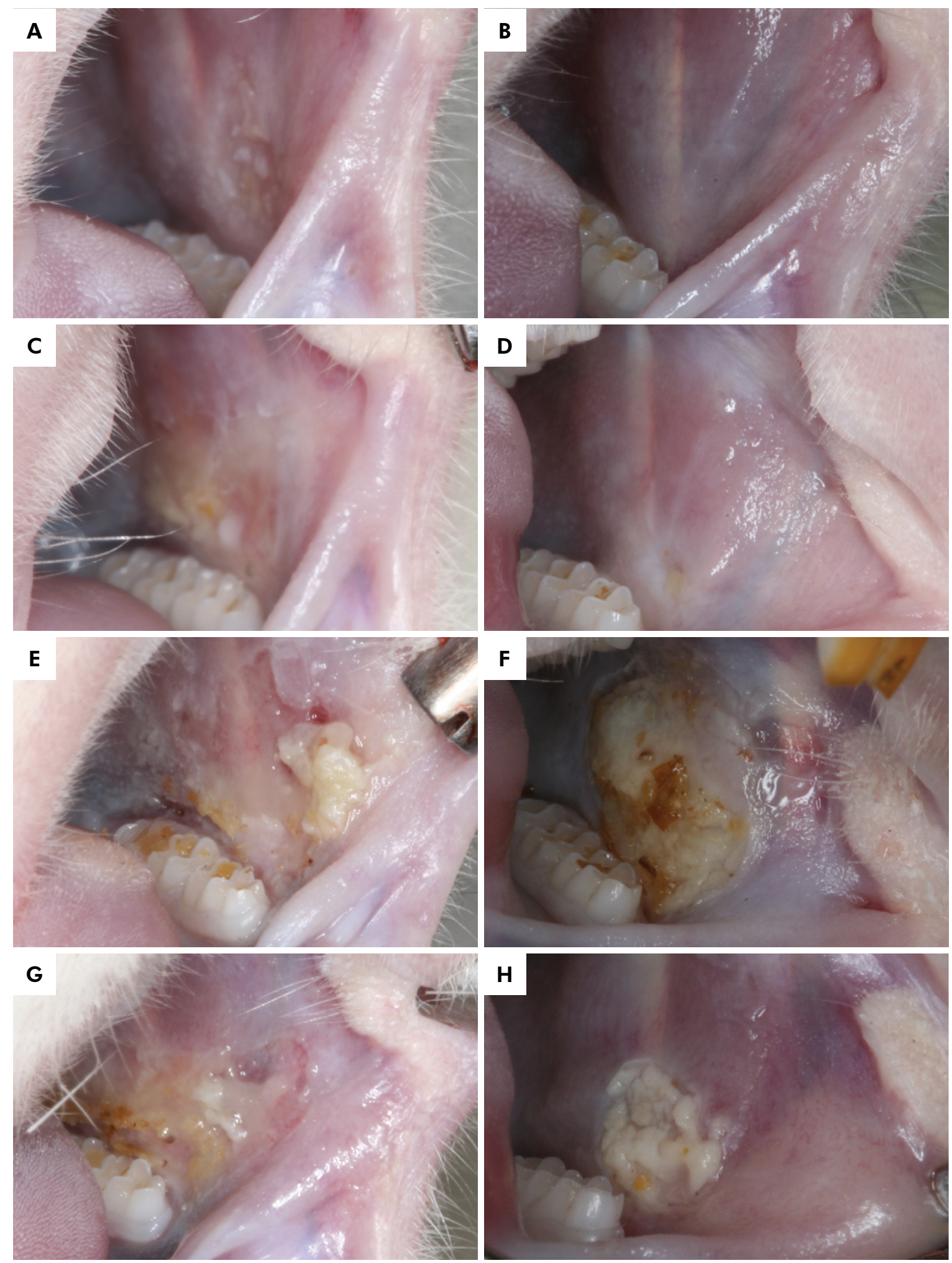

Figure 2. In groups GC and GPROB, the jugal mucosa presented discreet ulceration at day 3 ( $A$ and $C$ ), but was no longer observed at day 7 (B and D); groups G5-FU and G5-FU/PROB presented a single ulcerative lesion at day 3 (E and G), characterized as whitish, soft, of irregular shape and elevated edges, moderate erythema and hyperemia; at day 7, group G5-FU (F) presented a well-delimited lesion having severe erythema and hyperemia, and having increased in size from day 3 to 7; group G5-FU/PROB presented a reduction in the ulcerative lesion area from day $3(G)$ to $7(H)$, with no erythema or hyperemia. 
inflammatory infiltration, and mucosal ulceration covered by a fibrin-leukocyte crust at day 3 (Figure 3E), whereas extensive ulcerations and mucosal tissue necrosis covered by fibrin-leukocyte crust and bacterial colonies (Figure 3F) were observed at day 7. On the other hand, group G5-FU/PROB showed the prevalence of re-epithelialized areas and discreet inflammatory infiltration (Figure 3G) at day 3, and full mucosal re-epithelialization and moderate submucosal inflammatory infiltration at day 7 (Figure 3H). Comparison among the groups based on histological scores showed a statistically significant difference only between groups GPROB and G5-FU at day 7 (Table 1).

\section{Histomorphometric intestinal analysis}

Groups GC (Figures 4A and B) and GPROB (Figures $4 \mathrm{C}$ and D) presented normal intestinal structure. The animals that received chemotherapy presented moderate submucosal lymphocytic infiltration with destruction sites of villous surfaces at day 3 (G5-FU: Figure 4E; G5-FU/PROB: Figure 4G). At day 7, group G5-FU/PROB showed normal intestinal mucosa (Figure 4F), whereas group G5-FU presented discreet submucosal lymphocytic infiltration (Figure $4 \mathrm{H}$ ). The chemotherapy seems to have significantly decreased and damaged the intestinal villi of the animals. These changes were present to a lesser extent in the animals of group G5-FU/PROB.

The extensive tissue destruction observed in groups G5-FU and G5-FU/PROB precluded performing VH measurements in this groups. The mean and standard $\mathrm{VH}$ deviations in sections of the small intestines regarding groups GC and GPROB, as compared among the groups, are described in Table 2.

\section{Discussion}

The present study evaluated the effect of systemic administration of probiotics on the progression of experimentally induced oral and intestinal mucositis in rats. Clinical observation revealed regression of oral ulcerated lesions in group G5-FU/PROB, from day 3 to 7 , versus a progression of the lesions in group G5-FU, observed during the same period. Histologically, fewer severe signs of inflammation of oral mucosa were observed in group G5-FU/PROB than group G5-FU.
Table 1. Histological score of the jugal mucosa: count and percentage of animals classified according to the respective score $(n=3)$, with intergroup comparison.

\begin{tabular}{|c|c|c|c|c|c|c|}
\hline \multirow{2}{*}{ Time } & \multirow{2}{*}{ Group } & \multicolumn{4}{|c|}{ Score } & \multirow{2}{*}{ Total } \\
\hline & & 0 & 1 & 2 & 3 & \\
\hline \multirow{11}{*}{3 days } & \multirow{2}{*}{$\mathrm{GC}^{a}$} & 1 & 0 & 2 & 0 & 3 \\
\hline & & $33.3 \%$ & $0.0 \%$ & $66.7 \%$ & $0.0 \%$ & $100 \%$ \\
\hline & \multirow{2}{*}{$\mathrm{GPROB}^{a}$} & 2 & 0 & 1 & 0 & 3 \\
\hline & & $66.7 \%$ & $0.0 \%$ & $33.3 \%$ & $0.0 \%$ & $100 \%$ \\
\hline & \multirow{2}{*}{ G5-FUa } & 0 & 0 & 3 & 0 & 3 \\
\hline & & $0.0 \%$ & $0.0 \%$ & $100.0 \%$ & $0.0 \%$ & $100 \%$ \\
\hline & \multirow{2}{*}{$\begin{array}{l}\text { G5FU/ } \\
\text { PROB }^{\circ}\end{array}$} & 0 & 2 & 1 & 0 & 3 \\
\hline & & $0.0 \%$ & $66.7 \%$ & $33.3 \%$ & $0.0 \%$ & $100 \%$ \\
\hline & \multirow{2}{*}{ Total } & 3 & 2 & 7 & 0 & 12 \\
\hline & & $25.0 \%$ & $16.7 \%$ & $58.3 \%$ & $0.0 \%$ & $100 \%$ \\
\hline & p-value & & & 349 & & \\
\hline \multirow{11}{*}{7 days } & \multirow{2}{*}{$G C^{a b}$} & 2 & 0 & 1 & 0 & 3 \\
\hline & & $66.7 \%$ & $0.0 \%$ & $33.3 \%$ & $0.0 \%$ & $100 \%$ \\
\hline & \multirow{2}{*}{$\mathrm{GPROB}^{a}$} & 3 & 0 & 0 & 0 & 3 \\
\hline & & $100.0 \%$ & $0.0 \%$ & $0.0 \%$ & $0.0 \%$ & $100 \%$ \\
\hline & \multirow{2}{*}{ G5-FU } & 0 & 0 & 0 & 3 & 3 \\
\hline & & $0.0 \%$ & $0.0 \%$ & $0.0 \%$ & $100.0 \%$ & $100 \%$ \\
\hline & \multirow{2}{*}{$\begin{array}{l}\text { G5FU/ } \\
\text { PROB }^{a b}\end{array}$} & 2 & 1 & 0 & 0 & 3 \\
\hline & & $66.7 \%$ & $33.3 \%$ & $0.0 \%$ & $0.0 \%$ & $100 \%$ \\
\hline & \multirow{2}{*}{ Total } & 7 & 1 & 1 & 3 & 12 \\
\hline & & $58.3 \%$ & $8.3 \%$ & $8.3 \%$ & $25.0 \%$ & $100 \%$ \\
\hline & $p$-value & & & 39 & & \\
\hline
\end{tabular}

Different letters for the same experimental time indicate statistical difference among the groups (Kruskal-Wallis, $\mathrm{p}<0.05$ ).

Regarding the intestine, defects of lesser magnitude in the villi were observed in group G5-FU/PROB, compared with group G5-FU. Moreover, group GPROB showed higher villus height then group GC.

The present study used rats immunosuppressed with 5-FU to assess the effect of systemic probiotic administration on mucositis progression. Macroscopic and histopathological analyses showed that the treatment of rats with 5-FU, followed by mechanical trauma to the jugal mucosa, causes significant oral and intestinal mucositis. Regarding OM, these data corroborate those of previous studies, which showed similar aspects of OM induced by mechanical trauma in animals immunosuppressed with 5-FU. ${ }^{6,8,33}$ Additionally, this model reproduces major signs and symptoms observed in OM induced by chemotherapy 

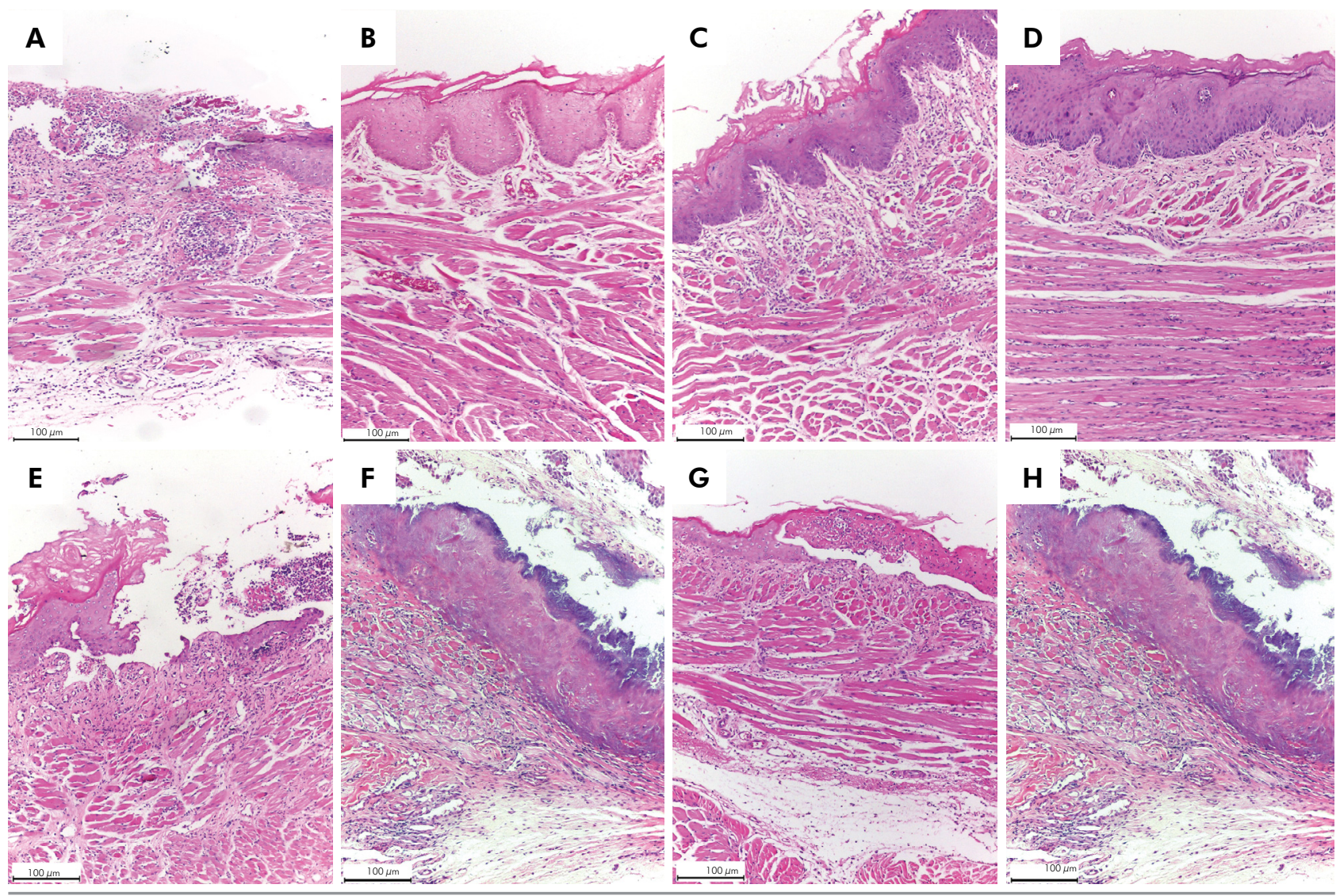

Figure 3. Light microscopy of the jugal mucosa. Group GC: A - Mucosal ulceration covered by a fibrin-leukocyte deposit; B - Full mucosal re-epithelialization and discreet submucosal inflammatory infiltration. Group GPROB: C and D - Unchanged epithelium. Group G5-FU: E - Mucosal ulceration covered by fibrin-leukocyte crust; F - Extensive mucosal ulceration covered by fibrin-leukocyte crust and bacterial colonies. Group G5-FU/PROB: G - Partial re-epithelialization of the epithelium with discreet stromal lymphocytic inflammatory infiltration; $\mathrm{H}$ - Full mucosal re-epithelialization and moderate submucosal inflammatory infiltration. The left column shows assessment at day 3, whereas the right column shows assessment at day 7 . Hematoxylin-eosin, 200x magnification.

in patients, and has been used extensively in the study of OM. The same pattern has also been observed for intestinal mucositis in the literature, ${ }^{19,21}$ which reports that 5-FU induces intestinal mucositis in rats.

Regarding feed consumption, measured weekly as of the beginning of the probiotic administration, there were no significant differences among the groups. These results indicate that the method of probiotic administration was effective, considering that its incorporation into the feed did not change its consumption. It is important to highlight that group G5-FU showed a drastic reduction in food intake from weeks 4 and 5, corresponding to postOM induction. Regarding animal weight, the only statistically significant difference among the groups was observed at day 7 , when group G5-FU presented the lowest body weight, in comparison with the other groups. The implication is that this reduced animal body weight may have been caused by the application of chemotherapy associated with the induction of $\mathrm{OM}$, not only because of the mechanical trauma to the mucosa, but also because of the systemic effects of the chemotherapy.

The macroscopic analysis of the oral mucosa showed that group G5-FU/PROB presented no erythema or hyperemia at day 7, whereas group G5-FU showed ulcerative lesion and severe erythema and hyperemia at the same time point. There was also a reduction in the ulcerative lesion area from day 3 to 7 in group G5-FU/PROB, whereas group G5-FU showed increased lesion size. These data were reinforced by the histopathological analysis. Therefore, the probiotic therapy reduced the inflammatory process in the rats 



Figure 4. Light microscopy of the small intestine. Groups GC (A and B) and GPROB (C and D) - Normal intestinal mucosa. Group G5-FU: E - Moderate submucosal lymphocytic infiltration with destruction of villous surfaces; F - Intestinal mucosa with discreet submucosal lymphocytic infiltration. Group G5-FU/PROB: G - Intestinal mucosa with moderate submucosal lymphocytic infiltration and areas with superficial villus destruction; $\mathrm{H}$ - Normal intestinal mucosa. The left column shows assessment at day 3 , whereas the right column shows assessment at day 7 . Hematoxylin-eosin, 100x magnification. 
Table 2. Mean and standard deviation $(\mu \mathrm{m})$ of villus height $(\mathrm{VH})$ of the small intestine (SI), with intra- and intergroup comparisons ( $\mathrm{n}=3$ ).

\begin{tabular}{cccc}
\hline Variable & 3 days & 7 days & p-value \\
\hline VH - SI & & & \\
GC & $194.8 \pm 116.3^{\mathrm{Aa}}$ & $222.4 \pm 7.5^{\mathrm{Ab}}$ & 0.022 \\
GPROB & $234.8 \pm 20.3^{\mathrm{Ba}}$ & $272.9 \pm 32.4^{\mathrm{Ba}}$ & $\mathrm{ns}$ \\
p-value & 0.034 & 0.055 & \\
\hline
\end{tabular}

Different uppercase letters $\mathrm{A}, \mathrm{B}$ indicate intergroup statistical difference (Student's ttest, $p<0.05)$; different lowercase letters ${ }^{a, b}$ indicate intragroup statistical difference (paired ttest, $\mathrm{p}<0.05$ ).

subjected to experimentally induced OM. In the present study, the probiotic therapy started 30 days before OM induction, as described by Messora et al..$^{31}$. This proved useful to determining the preventive effect of the therapy on the development and evolution of OM.

Considering the intestinal histomorphometric analysis, a major finding reports the protective effects of probiotic supplementation on the intestinal structure of animals. In this study, the animals from group GPROB showed greater villus height, compared to group GC. Moreover, normal intestinal mucosa was observed microscopically in the animals from group G5-FU/PROB at day 7, whereas group G5-FU showed signs of inflammation. This finding has already been reported by Smith et al., ${ }^{21}$ who observed that Lactobacillus fermentum was effective in reducing jejunal inflammation of the upper small intestine after 5-FU injection in rats. Whitford et al. ${ }^{19}$ also demonstrated that Streptococcus thermophiles reduces histological severity of intestinal mucositis in rats treated with 5-FU. Some mechanisms related to the probiotic used may have contributed to this result, such as the reduction in certain pro-inflammatory cytokines, activation of "natural killer" macrophages and lymphocytes, and stimulation of both immature leukocyte production and interferon production. ${ }^{35,36}$ Probiotics improve innate immunity and modulate pathogen-induced inflammation, leading to phagocytosis of the pathogen. It is important to emphasize that the intestinal ecosystem is partially responsible for maintaining human health. Specific changes in this ecosystem may contribute to the development of certain diseases. ${ }^{37}$

There is a considerable difference in the findings of studies that assess the effects of probiotics resulting from dose variations, treatment time, bacterial species and application methods. The high degree of species and strain specificity associated with the beneficial effects exerted by probiotics makes it difficult to understand the mechanisms underlying these effects completely. ${ }^{38}$ In the present study, a mix of Bacillus subtilis (2.9 $\left.\times 10^{8} \mathrm{UFC} / \mathrm{g}\right)$, Bifidobacterium bifidum $\left(2.0 \times 10^{8} \mathrm{UFC} / \mathrm{g}\right)$, Enterococcus faecium $\left(2.1 \times 10^{8} \mathrm{UFC} / \mathrm{g}\right)$ and Lactobacillus acidophilus $\left(1.0 \times 10^{8} \mathrm{UFC} / \mathrm{g}\right)$ was used. Although there are only a small number of studies evaluating the effect of probiotics on oral mucositis, the number of studies evaluating these effects on intestinal mucositis is somewhat greater; these indicate that the use of Lactobacillus fermentum $\left(1 \times 10^{6} \text { and } 1 \times 10^{9} \mathrm{CFU}\right)^{21}$ and Streptococcus thermophile $\left(6 \times 10^{9} \mathrm{CFU}\right)^{19}$ has been found to be effective.

Although probiotic therapy seems to be safe and risk-free, there is a small body of evidence of side effects related to systemic infections. ${ }^{39}$ It is also important to discuss the limitation of the sample in the present study. Considering that this is the first study evaluating the effects of probiotic administration on oral mucositis, and taking into account the restrictions on animal use, the authors thought it wise to use a reduced number of samples. Thus, more animal model studies and clinical trials should be developed to assess the safety and effectiveness of probiotics, before they are introduced as therapy in clinical practice.

\section{Conclusion}

Considering the limitations of the present study, it may be concluded that probiotic supplementation reduced oral and intestinal inflammation in immunosuppressed rats with experimentally induced mucositis, and may protect the intestine from changes induced by chemotherapy, thus contributing to overall health.

\section{Acknowledgements}

The authors would like to thank the Scholarships for Scientific Initiation Program (Probic) granted by Unoeste for its financial support. We also thank Alexandre Sant'ana de Oliveira, Mariana Fonseca Motta Borges and Talita Rizo Pereira, technicians of the Unoeste Pathological Anatomy Laboratory, for their technical support. 
- Probiotic therapy reduces inflammation and improves intestinal morphology in rats with induced oral mucositis

\section{References}

1. Diasio RB, Harris BE. Clinical pharmacology of 5-fluorouracil. Clin Pharmacokinet. 1989;16(4):215-37. https://doi.org/10.2165/00003088-198916040-00002

2. Sonis ST, Fey EG. Oral complications of cancer therapy. Oncology (Williston Park). 2002;16(5):680-6.

3. Albuquerque CL, Soares MSM, Silva DF. Mucosite oral: patobiologia, prevenção e tratamento. Comun Ciências Saúde. 2010;21(2):133-8.

4. Fischer DJ, Epstein JB. Management of patients who have undergone head and neck cancer therapy. Dent Clin North Am. 2008;52(1):39-60. https://doi.org/10.1016/i.cden.2007.09.004

5. Pico JL, Avila-Garavito A, Naccache P. Mucositis: its occurrence, consequences, and treatment in the oncology setting. Oncologist. 1998;3(6):446-51.

6. Sonis ST. Mucositis as a biological process: a new hypothesis for the development of chemotherapy-induced stomatotoxicity. Oral Oncol. 1998;34(1):39-43. https://doi.org/10.1016/S1368-8375(97)00053-5

7. Brown LR, Dreizen S, Handler S, Johnston DA. Effect of radiation-induced xerostomia on human oral microflora. J Dent Res. 1975;54(4):740-50. https://doi.org/10.1177/00220345750540040801

8. Epstein JB, Gorsky M, Guglietta A, Le N, Sonis ST. The correlation between epidermal growth factor levels in saliva and the severity of oral mucositis during oropharyngeal radiation therapy. Cancer. 2000;89(11):2258-65. https:// doi.org/10.1002/1097-0142(20001201)89:11<2258::AIDCNCR14>3.0.CO;2-Z

9. Bayer S, Kazancioglu HO, Acar AH, Demirtas N, Kandas NO Comparison of laser and ozone treatments on oral mucositis in an experimental model. Lasers Med Sci. 2017;32(3):673-7. https://doi.org/10.1007/s10103-017-2166-1

10. Volpato LER, Silva TC, Oliveira TM, Sakai VT, Machado MAAM. Radiation therapy and chemotherapy-induced oral mucositis. Rev Bras Otorrinolaringol. 2007;73(4):562-8. https://doi.org/10.1016/S1808-8694(15)30110-5

11. Keefe DMK, Gibson RJ, Hauer-Jensen M. Gastrointestinal mucositis. Semin Oncol Nurs. 2004;20(1):38-47. https://doi.org/10.1053/i.soncn.2003.10.007

12. Cool JC, Dyer JL, Xian CJ, Butler RN, Geier MS, Howarth GS. Pre-treatment with insulin-like growth factor-I partially ameliorates 5-fluorouracil-induced intestinal mucositis in rats. Growth Horm IGF Res. 2005;15(1):72-82. https://doi.org/10.1016/i.ghir.2004.12.002

13. Cummins AG, Jones BJ, Thompson FM. Postnatal epithelial growth of the small intestine in the rat occurs by both crypt fission and crypt hyperplasia. Dig Dis Sci. 2006;51(4):718-23. https://doi.org/10.1007/s10620-006-3197-9
14. Link KH, Kornmann M, Staib L, Redenbacher M, Kron M, Beger HG. Increase of survival benefit in advanced resectable colon cancer by extent of adjuvant treatment: results of a randomized trial comparing modulation of 5-FU + levamisole with folinic acid or with interferon-alpha. Ann Surg. 2005;242(2):178-87. https://doi.org/10.1097/01.sla.0000171033.65639.a9

15. Seiple L, Jaruga P, Dizdaroglu M, Stivers JT. Linking uracil base excision repair and 5 -fluorouracil toxicity in yeast. Nucleic Acids Res. 2006;34(1):140-51. https://doi.org/10.1093/nar/gki430

16. McCarthy GM, Awde JD, Ghandi H, Vincent M, Kocha WI. Risk factors associated with mucositis in cancer patients receiving 5-fluorouracil. Oral Oncol. 1998;34(6):484-90. https://doi.org/10.1016/S1368-8375(98)00068-2

17. Stringer AM, Gibson RJ, Logan RM, Bowen JM, Yeoh ASJ, Burns J et al. Chemotherapy-induced diarrhea is associated with changes in the luminal environment in the DA rat. Exp Biol Med (Maywood). 2007;232(1):96-106.

18. Gibson RJ, Keefe DMK. Cancer chemotherapy-induced diarrhoea and constipation: mechanisms of damage and prevention strategies. Support Care Cancer. 2006;14(9):890-900. https://doi.org/10.1007/s00520-006-0040-y

19. Whitford EJ, Cummins AG, Butler RN, Prisciandaro LD, Fauser JK, Yazbeck R et al. Effects of Streptococcus thermophilus $\mathrm{TH}-4$ on intestinal mucositis induced by the chemotherapeutic agent 5-Fluorouracil (5-FU). Cancer Biol Ther. 2009;8(6):505-11. https://doi.org/10.4161/cbt.8.6.7594

20. Mauger CA, Butler RN, Geier MS, Tooley KL, Howarth GS. Probiotic effects on 5-fluorouracil-induced mucositis assessed by the sucrose breath test in rats. Dig Dis Sci. 2007;52(3):612-9. https://doi.org/10.1007/s10620-006-9464-y

21. Smith CL, Geier MS, Yazbeck R, Torres DM, Butler RN, Howarth GS. Lactobacillus fermentum BR11 and fructo-oligosaccharide partially reduce jejunal inflammation in a model of intestinal mucositis in rats. Nutr Cancer. 2008;60(6):757-67. https://doi.org/10.1080/01635580802192841

22. Tang Y, Wu Y, Huang Z, Dong W, Deng Y, Wang $F$ et al. Administration of probiotic mixture DM\# 1 ameliorated 5-fluorouracil-induced intestinal mucositis and dysbiosis in rats. Nutrition. 2017;33:96-104 https://doi.org/10.1016/j.nut.2016.05.003

23. Yeung CY, Chan WT, Jiang CB, Cheng ML, Liu CY, Chang SW et al. Amelioration of chemotherapy-induced intestinal mucositis by orally administered probiotics in a mouse model. PLoS One. 2015;10(9):1-16. https://doi.org/10.1371/journal.pone.0138746

24. Vrese M, Schrezenmeir J. Probiotics, prebiotics, and synbiotics. Adv Biochem Eng Biotechnol. 2008;111:1-66. https://doi.org/10.1007/10_2008_097 
25. Saxelin M, Tynkkynen S, Mattila-Sandholm T, De Vos WM. Probiotic and other functional microbes: from markets to mechanisms. Curr Opin Biotechnol. 2005;16(2): 204-11. https://doi.org/10.1016/i.copbio.2005.02.003

26. Teughels W, Van Essche M, Sliepen I, Quirynen M. Probiotics and oral healthcare. Periodontol 2000. 2008;48(1):111-47. https://doi.org/10.1111/j.1600-0757.2008.00254.x

27. Lawande S. Probiotics for management of periodontal disease: a novel therapeutic strategy? IOSR J Pharm. 2012;2(4):41-6.

28. Meurman JH. Probiotics: do they have a role in oral medicine and dentistry? Eur J Oral Sci. 2005;113(3):188-96. https://doi.org/10.1111/j.1600-0722.2005.00191.x

29. Ahola AJ, Yli-Knuuttila H, Suomalainen T, Poussa T, Ahlström A, Meurman JH et al. Short-term consumption of probiotic-containing cheese and its effect on dental caries risk factors. Arch Oral Biol. 2002;47(11):799-804. https://doi.org/10.1016/S0003-9969(02)00112-7

30. Caglar E, Cildir SK, Ergeneli S, Sandalli N, Twetman S. Salivary mutans streptococci and lactobacilli levels after ingestion of the probiotic bacterium Lactobacillus reuteri ATCC 55730 by straws or tablets. Acta Odontol Scand. 2006;64(5):314-8. https://doi.org/10.1080/00016350600801709

31. Messora MR, Oliveira LFF, Foureaux RC, Taba M, Zangerônimo MG, Furlaneto FA et al. Probiotic therapy reduces periodontal tissue destruction and improves the intestinal morphology in rats with ligature-induced periodontitis. J Periodontol. 2013;84(12):1818-26. https://doi.org/10.1902/jop.2013.120644

32. Sonis ST, Tracey C, Shklar G, Jenson J, Florine D. An animal model for mucositis induced by cancer chemotherapy. Oral Surg Oral Med Oral Pathol. 1990;69(4):437-43. https://doi.org/10.1016/0030-4220(90)90376-4
33. Mitsuhashi H, Suemaru K, Li B, Cui R, Araki H. Evaluation of topical external medicine for 5 -fluorouracil-induced oral mucositis in hamsters. Eur J Pharmacol. 2006;551(1-3):152-5. https://doi.org/10.1016/i.ejphar.2006.09.005

34. Lima V, Brito GAC, Cunha FQ, Rebouças CG, Falcão BAA, Augusto RF et al. Effects of the tumour necrosis factor- $\alpha$ inhibitors pentoxifylline and thalidomide in short-term experimental oral mucositis in hamsters. Eur J Oral Sci. 2005;113(3):210-7. https://doi.org/10.1111/j.1600-0722.2005.00216.x

35. Park SY, Kim YH, Kim EK, Ryu EY, Lee SJ. Heme oxygenase-1 signals are involved in preferential inhibition of pro-inflammatory cytokine release by surfactin in cells activated with Porphyromonas gingivalis lipopolysaccharide. Chem Biol Interact. 2010;188(3):437-45. https://doi.org/10.1016/i.cbi.2010.09.007

36. Brunt J, Austin B. The development of probiotics for the control of multiple bacterial diseases of rainbow trout, Oncorhynchus mykiss (Walbaum). J Fish Dis. 2007;30(10):573-9. https://doi.org/10.1111/i.1365-2761.2007.00836.x

37. Prakash S, Rodes L, Coussa-Charley M, Tomaro-Duchesneau C, Tomaro-Duchesneau C, CoussaCharley. Gut microbiota: next frontier in understanding human health and development of biotherapeutics. Biologics. 2011;5:71-86. https://doi.org/10.2147/BTT.S19099

38. Prisciandaro LD, Geier MS, Butler RN, Cummins AG, Howarth GS. Evidence supporting the use of probiotics for the prevention and treatment of chemotherapy-induced intestinal mucositis. Crit Rev Food Sci Nutr. 2011;51(3):239-47. https://doi.org/10.1080/10408390903551747

39. Snydman DR. The safety of probiotics. Clin Infect Dis. 2008;46(Suppl 2):S104-11. https://doi.org/10.1086/523331 